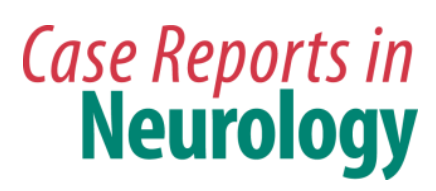

\title{
Emergency Superficial Temporal Artery to Middle Cerebral Artery Bypass after Intravenous Recombinant Tissue Plasminogen Activator Administration for Acute Cerebral Ischemia in a Patient with Moyamoya Disease
}

\author{
Sadaharu Tabuchia ${ }^{a}$ Sadao Nakajima ${ }^{a}$ Yutaka Suto $^{b}$ \\ Hiroyuki Nakayasu ${ }^{b}$ \\ Departments of a Neurosurgery and ${ }^{b}$ Neurology, Tottori Prefectural Central Hospital, \\ Tottori, Japan
}

\section{Key Words}

Acute cerebral ischemia - Acute revascularization - Tissue plasminogen activator - Moyamoya disease $\cdot$ Superficial temporal artery-middle cerebral artery anastomosis $\cdot$ Emergency bypass

\begin{abstract}
There are few study data to help in the decision whether to perform aggressive surgical revascularization, such as emergency bypass, after intravenous recombinant tissue plasminogen activator (rt-PA) administration in patients with progressive symptoms due to acute cerebral ischemia. A 33-year-old healthy male with no known previous medical history developed right hemiparesis and motor aphasia. No acute lesion was observed on admission computed tomography. According to the treatment protocol, emergency intravenous rt-PA administration was indicated within $3 \mathrm{~h}$. After rt-PA administration, symptoms progressed to complete right hemiplegia. Emergency magnetic resonance imaging (MRI) showed an acute ischemic lesion in the left basal ganglia. MR angiography showed severe stenosis of the bilateral terminal portion of the internal carotid artery and occlusion of the left middle cerebral artery (MCA). Obvious diffusion-perfusion mismatch was detected. We performed digital subtraction angiography and diagnosed this condition as acute cerebral ischemia
\end{abstract}

Sadaharu Tabuchi, MD, PhD

Department of Neurosurgery

Tottori Prefectural Central Hospital

730 Ezu, Tottori 680-0901 (Japan)

E-Mail tabuchis@pref.tottori.jp 
Tabuchi et al.: Emergency Superficial Temporal Artery to Middle Cerebral Artery Bypass after Intravenous Recombinant Tissue Plasminogen Activator Administration for Acute Cerebral Ischemia in a Patient with Moyamoya Disease

induced by moyamoya disease. We decided to perform emergency superficial temporal artery (STA)-MCA bypass to prevent further damage. The operation began $7 \mathrm{~h}$ after the administration of rt-PA and successful bypass was achieved. Symptoms stabilized and improved postoperatively. The majority of the area with preoperative hypoperfusion was rescued. Four months after surgery, the patient resumed his previous employment and continues to do well after 1.5 years of follow-up. This is the first report of emergency STAMCA bypass performed after intravenous rt-PA administration for acute cerebral ischemia in a patient with moyamoya disease. We conclude that emergency STA-MCA bypass is a viable option for patients with moyamoya disease even after administration of rt-PA.

(C) 2013 S. Karger AG, Basel

\section{Introduction}

Although treatment with intravenous recombinant tissue plasminogen activator (rt-PA) within $3 \mathrm{~h}$ of onset of ischemic stroke can improve clinical outcomes, some patients experience neurologic deterioration after the administration of rt-PA. There are few study data to help in the decision whether to perform aggressive surgical revascularization, such as emergency bypass, after rt-PA administration in patients with progressive symptoms due to acute cerebral ischemia. The present report describes the case of a patient with moyamoya disease who experienced neurologic deterioration after rt-PA administration for acute ischemic stroke and who subsequently underwent successful management with emergency superficial temporal artery (STA) to middle cerebral artery (MCA) bypass.

\section{Case Report}

A 33-year-old healthy male with no known previous medical history developed right hemiparesis and motor aphasia and was referred to our hospital. The patient's National Institutes of Health Stroke Scale (NIHSS) score was 20 points at the time of admission. A computed tomography (CT) scan was performed immediately and showed no acute lesion. According to the treatment protocol for acute cerebral ischemia, the patient received emergency intravenous rt-PA administration $(0.6 \mathrm{mg} / \mathrm{kg})$ by a neurologist at our hospital $2 \mathrm{~h}$ and 50 min after the onset of the event. After completion of rt-PA administration, symptoms progressed to complete right hemiplegia. During this treatment, the mean blood pressure was stable at $120-130 \mathrm{~mm} \mathrm{Hg}$, oxygen saturation was maintained at $>99 \%$ by oxygen inhalation (2 l/min), and blood glucose level was stable (109-113 mg/dl). Emergency magnetic resonance imaging (MRI) showed an acute ischemic lesion in the left basal ganglia to corona radiata (fig. 1a-c). MR angiography (MRA) showed severe stenosis of the bilateral terminal portion of the internal carotid artery (ICA) and occlusion at the origin of the left MCA (fig. 1d). Perfusion MRI showed marked prolongation of the mean transit time (MTT) in a broad area of the left MCA territory, including Broca's area, indicating the existence of a large area of hypoperfusion (fig. 2a). Obvious diffusion-perfusion mismatch was detected. Blood pressure values measured just before and after MRI were 129 and $144 \mathrm{~mm} \mathrm{Hg}$, respectively.

The patient was referred to our department for consideration of endovascular revascularization. We performed digital subtraction angiography, which showed mild stenosis of the terminal portion of the right ICA, severe stenosis of the A1 portion of the right anterior cerebral artery, and severe stenosis of the terminal portion of the left ICA and M1 portion of 
Tabuchi et al.: Emergency Superficial Temporal Artery to Middle Cerebral Artery Bypass after Intravenous Recombinant Tissue Plasminogen Activator Administration for Acute Cerebral Ischemia in a Patient with Moyamoya Disease

the left MCA, with poor peripheral circulation when compared with the right (fig. 1e, f). Based on these data, acute cerebral ischemia induced by moyamoya disease or moyamoyalike angiopathy was diagnosed, because the patient had no remarkable past medical history or risk factors for arteriosclerosis. We first considered emergency endovascular revascularization because of clinical progression despite best medical treatment, but in a second stage, we considered the STA-MCA bypass was a better option than endovascular ballooning or stenting for this case on the premise of underlying moyamoya disease and progressive angiopathy. Since the patient showed no significant improvement during the examination, we finally decided to perform emergency STA-MCA bypass to prevent further progression of cerebral ischemia.

Detailed informed consent was obtained from the patient's family, and the operation began $7 \mathrm{~h}$ after the administration of rt-PA. A successful single bypass using the parietal branch of the STA with a small craniotomy was achieved, as demonstrated by intraoperative indocyanine green videoangiography (data not shown). Revascularization was accomplished $9.5 \mathrm{~h}$ after the administration of rt-PA. Symptoms stabilized and subsequently improved postoperatively. The free radical scavenger edaravone $(60 \mathrm{mg} /$ day) [1] was given postoperatively to minimize ischemic damage. Follow-up CT was performed on postoperative day 2 and confirmed the patency of the bypass, absence of postoperative hemorrhage, and no apparent progression of infarction. The phosphodiesterase inhibitor and antiplatelet and vasodilating agent cilostazol (200 mg/day) [2] was started by oral administration on postoperative day 2 to improve peripheral cerebral blood flow and to reduce edema formation. MRI was performed on postoperative day 4 and confirmed the patency of the bypass with persistent stenosis of the terminal portion of bilateral ICA (fig. 3a) and new small lesions when compared with those seen on preoperative diffusion-weighted imaging (DWI) (fig. 3b). Perfusion MRI showed complete recovery of MTT in the left cerebral hemisphere (fig. 2b). Most areas of preoperative hypoperfusion seen after rt-PA administration were rescued by the emergency operation and additional medical treatment. Blood pressure values measured just before and after MRI were 113 and $108 \mathrm{~mm} \mathrm{Hg}$, respectively.

Hyperperfusion syndrome and hemorrhage into an area of ischemia did not occur. Four months after surgery, the patient resumed his previous full-time employment (Department of Statistical Analysis) and continued to take cilostazol (200 mg/day). He remains well at the 1.5-year follow-up visit.

\section{Discussion}

Although treatment with intravenous rt-PA within $3 \mathrm{~h}$ of onset of ischemic stroke can improve clinical outcome according to the rt-PA stroke study conducted by the National Institute of Neurological Disorders and Stroke (NINDS) in 1995 and a study by the Japan Alteplase Clinical Trial (J-ACT) group in 2006, some patients can experience neurologic deterioration after the administration of rt-PA. In such cases, surgical revascularization (e.g. endovascular surgery, carotid endarterectomy, and STA-MCA bypass) is sometimes considered. However, additional antiplatelet therapy, anticoagulation therapy, and invasive surgical treatment should generally be avoided or require special attention when performed within $24 \mathrm{~h}$ after the administration of rt-PA, as the fibrinolytic system is stimulated for approximately $24 \mathrm{~h}$, even though the half-life of rt-PA is $84.2 \mathrm{~min}$.

Because extracranial-intracranial bypass is performed infrequently at most hospitals, experience with emergency surgical bypass has been limited. Several studies have described the safety and efficacy of emergency STA-MCA bypass for acute ischemic patients presenting 
with progressive symptoms despite best medical treatment [3-5]. The efficacy and safety of emergency STA-MCA bypass within $24 \mathrm{~h}$ after the administration of rt-PA have not been well characterized, although one recent report presented successful results when using this strategy [6]. Those investigators performed emergency bypass in patients with ICA occlusion $9 \mathrm{~h}$ after the administration of rt-PA. The present case was complicated by the presence of moyamoya disease. Although patients with moyamoya disease require special attention when given rt-PA according to the revised Japanese guideline for the management of stroke 2012, our patient had not been diagnosed with moyamoya disease until after rt-PA administration. Several reports have described primary percutaneous angioplasty and stenting for the prevention of ischemia in patients with moyamoya disease. One report concluded that the therapeutic effect of angioplasty and stenting was not durable and did not provide long-term prevention against future ischemic events in patients with moyamoya disease [7]. Another study reported that delayed severe intracranial hemorrhage occurred after intracranial carotid artery stent placement in a patient with moyamoya disease [8]. In the present case, emergency STA-MCA bypass resulted in excellent outcomes in a patient with moyamoya disease who experienced neurologic deterioration after rt-PA administration for acute ischemic stroke.

\section{Conclusion}

This is the first report of emergency STA-MCA bypass performed $7 \mathrm{~h}$ after intravenous rt-PA administration for acute cerebral ischemia in a patient with moyamoya disease. We conclude that emergency STA-MCA bypass is a viable option for patients with moyamoya disease who experienced progression of ischemic symptoms after administration of rt-PA for acute ischemic stroke.

\section{Disclosure Statement}

The authors have no personal financial or institutional interest in any of the drugs, materials, or devices mentioned in the article.

\section{References}

1 Shinohara Y, Saito I, Kobayashi S, Uchiyama S: Edaravone (radical scavenger) versus sodium ozagrel (antiplatelet agent) in acute noncardioembolic ischemic stroke (EDO trial). Cerebrovasc Dis 2009;27:485492.

2 Shinohara Y, Katayama Y, Uchiyama S, Yamaguchi T, Handa S, Matsuoka K, et al: Cilostazol for prevention of secondary stroke (CSPS2): an aspirin-controlled, double-blind, randomized non-inferiority trial. Lancet Neurol 2010;9:959-968.

-3 Nussbaum ES, Janjua TM, Defillo A, Lowary JL, Nussbaum LA: Emergency extracranial-intracranial bypass surgery for acute ischemic stroke. J Neurosurg 2010;112:666-673.

-4 Sakai K, Nitta J, Horiuchi T, Ogiwara T, Kobayashi S, Tanaka Y, et al: Emergency revascularization for acute main-trunk occlusion in the anterior circulation. Neurosurg Rev 2008;31:69-76.

$\checkmark 5$ Yoshimoto Y, Kwak S: Superficial temporal artery-middle cerebral artery anastomosis for acute cerebral ischemia: the effect of small augmentation of blood flow. Acta Neurochir (Wien) 1995;137:128-137.

6 Ishishita Y, Kimura T, Morita A: Urgent superficial temporal artery to middle cerebral artery bypass shortly after intravenous rt-PA. Br J Neurosurg 2012;26:773-775.

-7 Khan N, Dodd R, Marks MP, Bell-Stephens T, Vavao J, Steinberg GK: Failure of primary percutaneous angioplasty and stenting in the prevention of ischemia in moyamoya angiopathy. Cerebrovasc Dis 2011;31:147-153. 


\begin{tabular}{l|l}
\hline Case Rep Neurol 2013;5:214-219 \\
\hline DOI: $10.1159 / 000357664$ & $\begin{array}{l}\text { C 2013 S. Karger AG, Basel } \\
\text { www.karger.com/crn }\end{array}$ \\
\hline
\end{tabular}

Tabuchi et al.: Emergency Superficial Temporal Artery to Middle Cerebral Artery Bypass after Intravenous Recombinant Tissue Plasminogen Activator Administration for Acute Cerebral Ischemia in a Patient with Moyamoya Disease

8 Eicker S, Etminan N, Turowski B, Steiger HJ, Hanggi D: Intracranial carotid artery stent placement causes delayed severe intracranial hemorrhage in a patient with moyamoya disease. J Neurointerv Surg 2011;3:160-162.
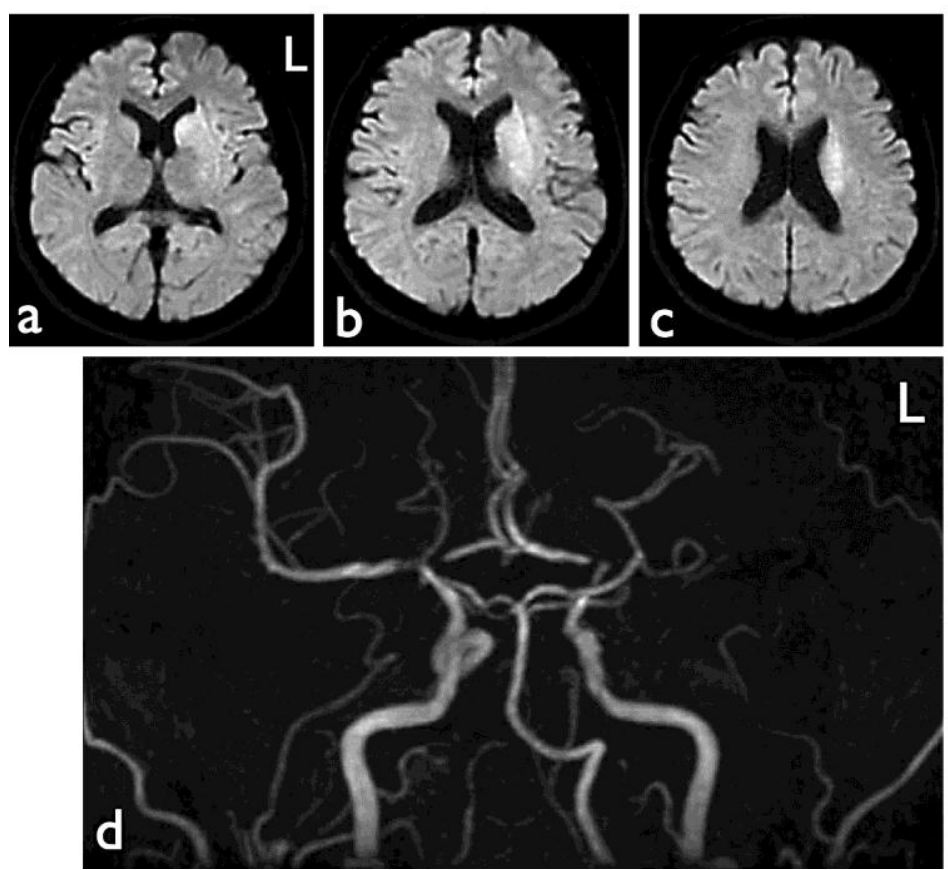
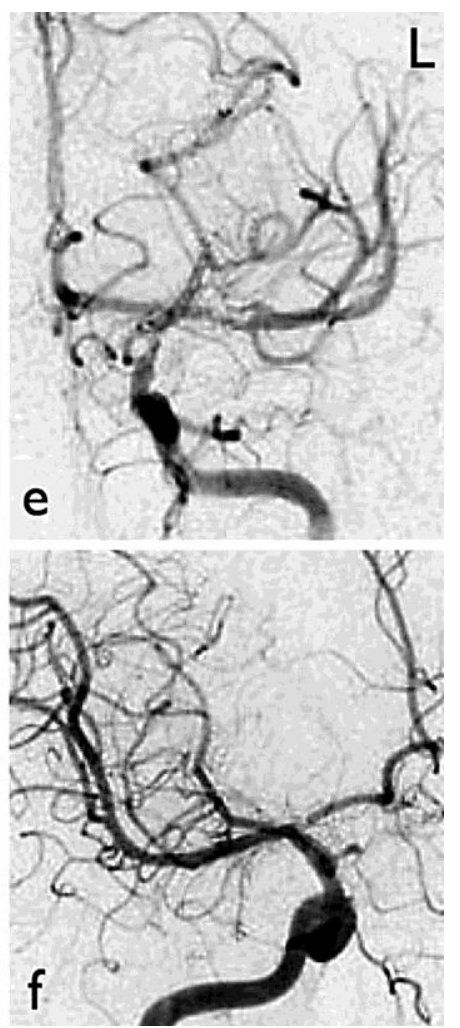

Fig. 1. DWI after the administration of rt-PA shows an acute ischemic lesion in the left basal ganglia to corona radiate $(\mathrm{a}-\mathrm{c})$. MRA shows severe stenosis of the bilateral terminal portion of the ICA and occlusion of the left MCA (d). Left carotid angiography shows severe stenosis of the terminal portion of the ICA and of the M1 portion of the MCA and poor peripheral flow (e). Right carotid angiography shows stenosis of the terminal portion of the ICA and severe stenosis of the A1 portion of the anterior cerebral artery (f). 


\section{Case Reports in Neurology}

\section{Case Rep Neurol 2013;5:214-219}

DOI: $10.1159 / 000357664$

Tabuchi et al.: Emergency Superficial Temporal Artery to Middle Cerebral Artery Bypass after Intravenous Recombinant Tissue Plasminogen Activator Administration for Acute Cerebral Ischemia in a Patient with Moyamoya Disease
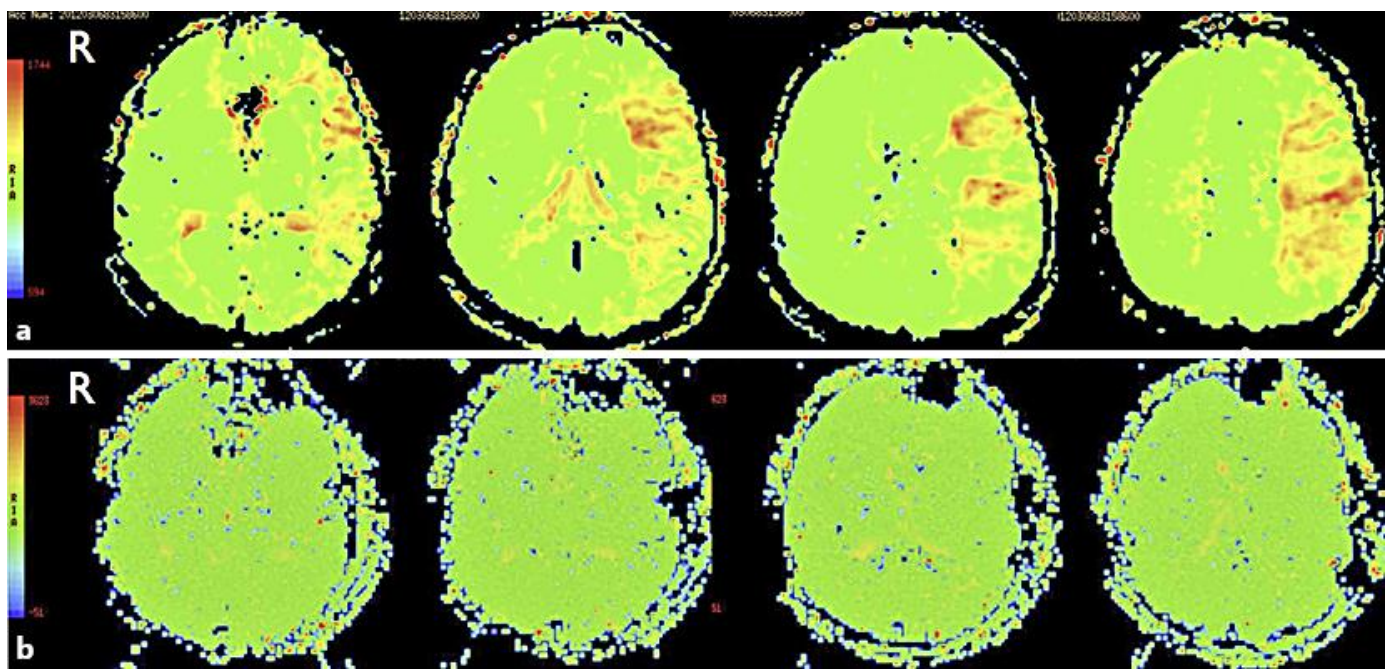

Fig. 2. Preoperative perfusion MRI shows that MTT was markedly prolonged in a broad area of the left hemisphere, including Broca's area (a). Postoperative perfusion MRI shows complete recovery of MTT in the left hemisphere (b).
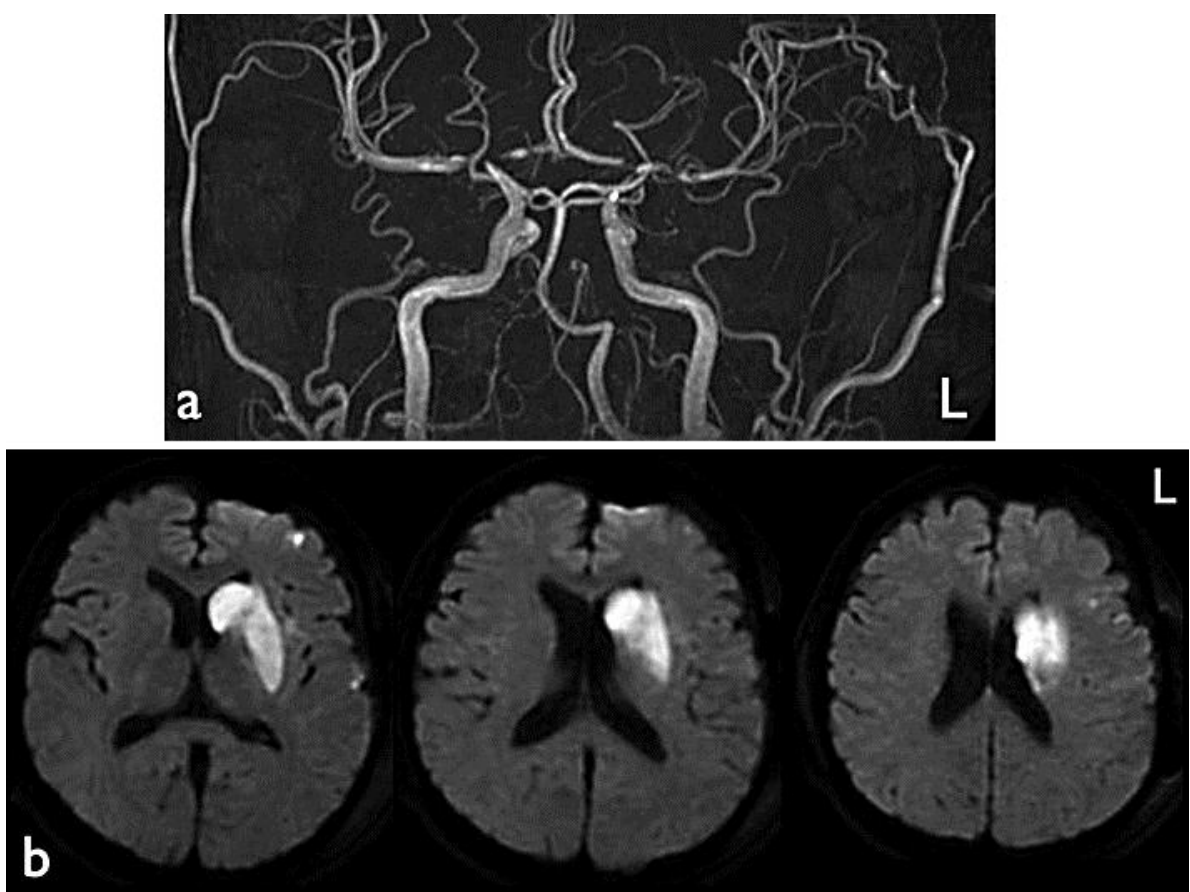

Fig. 3. Postoperative MRA shows patency of the bypass, good visualization of the left MCA, and persistent stenosis of the bilateral terminal portion of the ICA (a). Postoperative DWI shows new small lesions when compared with the preoperative image. This area is the final area of infarction (b). 